\title{
Konstruktion von Differenz : ein Vergleich argumentativer Strategien der Befürworter der Schächtverbotsinitiative (1893) und der Antiminarettinitiative (2009)
}

\author{
Autor(en): Metzger, Thomas \\ Objekttyp: Article \\ Zeitschrift: Schweizerische Zeitschrift für Religions- und Kulturgeschichte = \\ Revue suisse d'histoire religieuse et culturelle = Rivista svizzera di \\ storia religiosa e culturale
}

Band (Jahr): 112 (2018)

PDF erstellt am: $\quad \mathbf{0 6 . 0 3 . 2 0 2 0}$

Persistenter Link: http://doi.org/10.5169/seals-842404

\section{Nutzungsbedingungen}

Die ETH-Bibliothek ist Anbieterin der digitalisierten Zeitschriften. Sie besitzt keine Urheberrechte an den Inhalten der Zeitschriften. Die Rechte liegen in der Regel bei den Herausgebern.

Die auf der Plattform e-periodica veröffentlichten Dokumente stehen für nicht-kommerzielle Zwecke in Lehre und Forschung sowie für die private Nutzung frei zur Verfügung. Einzelne Dateien oder Ausdrucke aus diesem Angebot können zusammen mit diesen Nutzungsbedingungen und den korrekten Herkunftsbezeichnungen weitergegeben werden.

Das Veröffentlichen von Bildern in Print- und Online-Publikationen ist nur mit vorheriger Genehmigung der Rechteinhaber erlaubt. Die systematische Speicherung von Teilen des elektronischen Angebots auf anderen Servern bedarf ebenfalls des schriftlichen Einverständnisses der Rechteinhaber.

\section{Haftungsausschluss}

Alle Angaben erfolgen ohne Gewähr für Vollständigkeit oder Richtigkeit. Es wird keine Haftung übernommen für Schäden durch die Verwendung von Informationen aus diesem Online-Angebot oder durch das Fehlen von Informationen. Dies gilt auch für Inhalte Dritter, die über dieses Angebot zugänglich sind. 


\section{Konstruktion von Differenz: \\ Ein Vergleich argumentativer Strategien \\ der Befürworter der Schächtverbotsinitiative (1893) \\ und der Antiminarettinitiative (2009)}

Thomas Metzger

1891 erweiterte der Schweizerische Bundesstaat die direktdemokratischen Betätigungsmöglichkeiten und nahm das Initiativrecht in seine Verfassung auf. Zwei Jahre später gelangte mit der Schächtverbotsinitiative ein erstes Volksbegehren zur Abstimmung. Mit deutlich über 80`000 Unterschriften hatten die Initianten die damals verlangten $50^{\circ} 000$ Unterschriften mühelos übertroffen. ${ }^{1}$ Die Stimmberechtigten nahmen das Verbotsbegehren, das von den deutschschweizerischen Tierschutzvereinen lanciert worden war, nach einem stark antisemitisch geprägten Abstimmungskampf deutlich an. Das neue Volksrecht hatte somit bereits seine 〈Unschuld〉 verloren und war zu einem Instrument gesellschaftlicher Exklusion geworden, mit dessen Hilfe die Mehrheit die kleine religiöse Minderheit der Juden nur zwei Jahrzehnte nach deren voller Emanzipation erneut rechtlich und letztlich auch gesellschaftlich zurücksetzte. Mehr als hundert Jahre später, 2009, gelangte mit der Antiminarettinitiative ein weiteres Volksbegehren zur Abstimmung, welches die gezielte Ausgrenzung eines Teils der schweizerischen Bevölkerung beabsichtigte. Nun standen nicht mehr 〈die Juden〉, sondern 〈die Muslime> im Visier der Initianten, die sich aus Exponenten der rechtspopulistischen Schweizerischen Volkspartei (SVP) und der evangelikal-nationalkonservativen Kleinpartei Eidgenössische Demokratische Union (EDU) rekrutierten. Auch diese Initiative wurde durch Volk und Stände angenommen.

In der vergleichenden Analyse der Argumentationsstrategien der Befürworter der beiden Initiativbegehren, die zentrale Denkweisen und Sprachregelungen dieser Akteure aufzeigt, manifestieren sich interessante Parallelen. Diese sollen

1 Friedrich Traugott Külling, Bei uns wie überall? Antisemitismus in der Schweiz 18661900, Zürich [1977], 283. 1977 wurde die verlangte Unterschriftenanzahl auf $100^{`} 000$ verdoppelt. Siehe: Bernhard Degen, Art. (Volksinitiative〉, in: Historisches Lexikon der Schweiz, in: 〈www.hls-dhs-dss.ch/textes/d/D10386.php〉 (15. April 2018).

SZRKG, 112 (2018), 347-363 
im Zentrum der folgenden Ausführungen stehen. Quellengrundlage bilden zum einen Publikationen aus dem Umfeld der Initianten und zum anderen Beiträge aus der Zeitungsberichterstattung. ${ }^{2}$ Ein besonderes Augenmerk wird auf Differenzkonstruktionen gelegt, die in den Topoi und Diskursen der Verbotsbefürworter aufscheinen. ${ }^{3}$ Diese stilisierten 〈die Juden〉 und 〈die Muslime〉 in dichotomisierender Weise zu Bevölkerungsgruppen, die den als christlich apostrophierten 〈Schweizern〉 fremd gegenüberstehen würden. So ist es paradigmatisch, dass die Abstimmungen nicht zuletzt nationalistisch aufgeladen und mit Fragen wie Immigration und Integration verknüpft wurden. Die antisemitischen respektive islamfeindlichen Dimensionen der beiden angenommenen Volksinitiativen überlagern sich somit teilweise mit fremdenfeindlichen Diskursen. ${ }^{4}$

In den letzten Jahren ist gerade im deutschsprachigen Raum eine kontrovers geführte Diskussion um die Vergleichbarkeit von Antisemitismus und Islamfeindlichkeit entstanden. ${ }^{5}$ Der Vergleich als eine klassische historische Methode stösst auf inhaltlicher Ebene bei diesen Phänomenen schnell an Grenzen, zumal ihre historischen Entstehungszusammenhänge und Wirkmächtigkeit grosse Unterschiede aufweisen. Auf der Ebene argumentationsstruktureller Prinzipien, Funktionen und Vorurteilsmustern erscheint er hingegen fruchtbarer. ${ }^{6}$

Es fand keine systematische Untersuchung von Zeitungen statt. Die Resultate werden mit Erkenntnissen aus bestehenden Forschungsstudien ergänzt.

3 Für die Differenzkonstruktionen zugrundeliegenden Prozesse der Konstruktion von Identität und Alterität siehe aus der umfangreichen Forschungsliteratur: Anna Margaretha Horatschek/Anja Pistor-Hatam, Einleitung, in: dies. (Hg.), Identitäten im Prozess. Region, Nation, Staat, Individuum, Berlin/Boston 2016, 1-12; Robert Neisen, Feindbild, Vorbild, Wunschbild. Eine Untersuchung zum Verhältnis von britischer Identität und französischer Alterität 1814-1860, Würzburg 2004, v.a. 11-28.

4 Ich ziehe die Begriffe 〈Islamfeindlichkeit〉 und 〈Fremdenfeindlichkeit〉 den pathologisierenden und somit potenziell verharmlosenden Bezeichnungen 〈Islamophobie〉 und 〈Xenophobie> vor. In der noch jungen Forschung zur Islamfeindlichkeit besteht ein Nebeneinander verschiedener Begriffe, die kontrovers diskutiert werden. Für einen kurzen Überblick: Fabian Virchow, Islamkritik - Islamophobie - Antimuslimismus - Antimuslimischer Rassismus. Zur Begrifflichkeit eines viel diskutierten Phänomens, in: Hans-Peter Killguss/ Alexander Häusler (Hg.), Das Geschäft mit der Angst. Rechtspopulismus, Muslimfeindlichkeit und die extreme Rechte in Europa, Köln 2012, 15-19.

5 Entzündet hatte sich die Kontroverse an der vom Zentrum für Antisemitismusforschung in Berlin am 8. Dezember 2008 durchgeführten Tagung «Feindbild Muslim - Feindbild Jude». Im Zentrum der Kritik stand insbesondere Wolfgang Benz, der allerdings keineswegs für eine Gleichsetzung von Islamfeindlichkeit und Antisemitismus plädierte. Für die Resultate der Tagung siehe: Wolfgang Benz (Hg.), Islamfeindschaft und ihr Kontext. Dokumentation der Konferenz «Feindbild Muslim - Feindbild Jude», Berlin 2009. Einen breiteren Einblick bezüglich unterschiedlicher Haltungen gegenüber der Vergleichbarkeit bietet: Gideon Botsch/Olaf Glöckner/Christoph Kopke/Michael Spieker (Hg.), Islamophobie und Antisemitismus - ein umstrittener Vergleich, Berlin/Boston 2012.

6 Siehe auch die Argumentation von: Juliane Wetzel, Parallelen zwischen Antisemitismus und Islamfeindschaft heute, in: Botsch/Glöckner/Kopke/Spieker (Hg.), Islamophobie (wie Anm. 5), 81-106, 82-83. 
Ausgehend von einer historischen Einordnung der beiden Initiativbegehren und einer Charakterisierung der Abstimmungsresultate werden die Ergebnisse der vergleichenden Analyse von Quellen der Schächt- und der Minarettgegner ausgeführt. Dabei lassen sich die Diskurse bündeln: erstens die Verneinung der kultischen Relevanz des Schächtens und der Minarette; zweitens die Diskreditierung des Judentums und des Islam als «rückständige〉 und (gewaltbereite〉 Religionen; drittens das Beharren auf Statusdifferenz und viertens die explizite Stigmatisierung von Juden und Muslimen als «Fremde〉.

\section{Das Schächt- und das Minarettverbot als konfessionelle Ausnahmeartikel}

Die beiden religiösen Gruppen ausgrenzenden Bestimmungen, die aufgrund der erfolgreichen Volksinitiativen in die Schweizerische Bundesverfassung aufgenommen wurden, lassen sich in eine längere Tradition konfessioneller Ausnahmeartikel einreihen, welche in den ersten Jahrzehnten nach der Gründung des Schweizerischen Bundesstaates im Jahr 1848 installiert worden waren. Diese richteten sich vor dem Hintergrund des Bürgerkrieges von 1847 und dem Kulturkampf im letzten Drittel des 19. Jahrhunderts gegen den katholischen Bevölkerungsteil. Seit 1848 enthielt die Bundesverfassung ein Verbot des Jesuitenordens, zu welchem sich seit der Totalrevision von 1874 ein solches der Neugründung oder Wiederherstellung von Klöstern gesellte. Ebenso wurde in jener Totalrevision die Gründung neuer Bistümer von der Zustimmung des Bundes abhängig gemacht und somit dem Kompetenzbereich der katholischen Kirche entzogen. ${ }^{7}$ Im Gegensatz zum Schächt- und Minarettverbot wurden diese Artikel jedoch nicht durch das direktdemokratische Instrument der Volksinitiative in die Bundesverfassung aufgenommen. Als diese Artikel - zusammen mit dem fehlenden Frauenstimmrecht - der Ratifizierung der Europäischen Menschenrechtskonvention durch die Schweiz im Wege standen, wurde die Mehrheit der Artikel 1973 aus der Bundesverfassung gestrichen, nicht ohne dass in den Abstimmungen erneut alte Ressentiments geschürt worden wären. ${ }^{8}$ Das Schächtverbot

7 Zu den konfessionellen Ausnahmeartikeln siehe: Marco Jorio, Art 〈Ausnahmeartikel), in: Historisches Lexikon der Schweiz, in: 〈www.hls-dhs-dss.ch/textes/d/D10388.php〉 (20. April 2018); Adrian Vatter, Synthese: religiöse Minderheiten im direktdemokratischen System der Schweiz, in: ders. (Hg.), Vom Schächt- zum Minarettverbot. Religiöse Minderheiten in der direkten Demokratie, Zürich 2011, 264-290.

Zur Problematik der Ratifizierung der Europäischen Menschenrechtskonvention: Stefanie Tamara Kurt, Die rechtliche Situation von religiösen Minderheiten im Kontext des schweizerischen Föderalismus. Die Religionsfreiheit und das Verbot der religiösen Diskriminierung. Grundlagen - Konsequenzen - Perspektiven, Basel 2018, 43. Zur Persistenz antikatholischer Ressentiments: Vatter, Synthese (wie Anm. 7), 273-275. 2001 wurde mit dem sogenannten Bistumsartikel der letzte Ausnahmeartikel aufgehoben, der die Katholiken betraf. 
hingegen wurde nicht ersatzlos gestrichen, sondern aus der Bundesverfassung ins Tierschutzgesetz verschoben, womit es weiterhin Gültigkeit besitzt. ${ }^{9}$

Das Schächtverbot, das heute nebst Juden gerade auch für Muslime eine Einschränkung darstellt, sowie das Minarettverbot zeigen auf, dass die direkte Demokratie der Schweiz zur Aufnahme von Artikeln führen kann, die dem Diskriminierungsverbot und der ebenfalls durch die Verfassung garantierte Religionsfreiheit, die zugleich durch internationale Abkommen (Europäische Menschenrechtskonvention, zweiter UNO-Menschenrechtspakt) abgesichert sind, widersprechen. ${ }^{10}$ Überblickt man die Abstimmungsergebnisse der beiden Volksabstimmungen, so finden sich nur beschränkt Übereinstimmungen im Abstimmungsverhalten. Die Markanteste ist, dass beide Initiativen in der frankophonen Westschweiz abgelehnt wurden. Das Minarettverbot wurde insbesondere von rechtskonservativen Kreisen unterstützt, wusste aber auch in der politischen Mitte zu punkten. Liberale oder konservative Werthaltungen waren für das Stimmverhalten entscheidend. ${ }^{11}$ Dies führte am 29. November $2009 \mathrm{zu}$ einem 57,5 Prozent Ja-Stimmen-Anteil für die Antiminarettinitiative und zu einem klaren 19,5 zu 3,5 der Stände, deren Mehr für die Annahme einer Initiative ebenfalls Voraussetzung ist. ${ }^{12}$ Das Schächtverbot wurde am 20. August 1893 mit 60,1 Prozent angenommen. Mit 11,5 zu 10,5 fiel das Ständemehr hingegen denkbar knapp aus. ${ }^{13}$ Bei der Abstimmung von 1893 zeigte sich ein uneinheitlicheres Bild. Die Zustimmung in städtischen und oder protestantisch geprägten Gebieten der Deutschschweiz war deutlicher als in konservativ geprägten katholischen Landkantonen, da sich dort die Furcht davor zeigte, als Katholiken selbst erneut Opfer konfessioneller Ausnahmebestimmungen zu werden. Insgesamt jedoch war auch auf katholischer Seite das Bild uneinheitlich. ${ }^{14}$ Während beispielsweise der Kanton Nidwalden klar Nein sagte, stimmte der Nachbarkanton Obwalden der Initiative zu. Wichtig für den Abstimmungsausgang in den ein-

10

11

Siehe hierzu: Pascal Krauthammer, Das Schächtverbot in der Schweiz. Die Schächtfrage zwischen Tierschutz, Politik und Fremdenfeindlichkeit, Zürich 2000, 195-262.

Christoph Winzeler, Das Minarettverbot - eine Nachlese, in: ders., Religion im demokratischen Staat. Beiträge zum Religionsverfassungsrecht und zur Religionsfreiheit, Zürich/Basel/Genf 2012, 205-212, 206; Kurt, Die rechtliche Situation (wie Anm. 8), 24-27; 53-54.

Für eine differenzierte Abstimmungsanalyse siehe: Adrian Vatter/Thomas Milic/Hans Hirter, Das Stimmverhalten bei der Minarettverbots-Initiative unter der Lupe, in: Vatter (Hg.), Vom Schächt- zum Minarettverbot (wie Anm. 7), 144-170. Anna Christmann/Deniz Danaci/Oliver Krömler, Ein Sonderfall? Das Stimmverhalten bei der Minarettverbots-Initiative im Vergleich zu andern Abstimmungen und Sachfragen, in: Vatter (Hg.), Vom Schächtzum Minarettverbot (wie Anm. 7), 171-190; Klaus Stüwe, «A Kinder and Gentler Democracy»? Das Konsensusmodell der Schweiz und das Minarettverbot, in: Anton Rauscher (Hg.), Toleranz und Menschenwürde, Berlin 2011, 451-471.

〈www.bk.admin.ch/ch/d/pore/va/20091129/det547.html〉, (20. April 2018).

〈www.bk.admin.ch/ch/d/pore/va/18930820/det40.html), (20. April 2018).

Uneinheitlich traten auch die protestantischen Periodika der Deutschschweiz auf. Dies traf sowohl auf liberal- als auch konservativ-protestantische Zeitschriften zu. Siehe hierzu: Thomas Metzger, Antisemitismus im Deutschschweizer Protestantismus, Berlin 2017, 214-222. 
zelnen Kantonen waren letztlich 1893 insbesondere die lokalen Medien und Tierschutzvereine. ${ }^{15}$ Unterstützten diese ein Schächtverbot, durfte ein Ja erwartet werden. Wenn aber etwa wie im Kanton St. Gallen der kantonale Tierschutzverein nicht an der Kampagne seines Dachvereins partizipierte, konnte dies zu einem wuchtigen Nein führen. ${ }^{16}$

\section{Was 〈Kultus〉 ist, bestimmt die Mehrheitsgesellschaft}

Die Initianten sahen sich sowohl 1893 als auch 2009 von gegnerischer Seite mit dem Vorwurf konfrontiert, mit ihren Volksbegehren einen offensichtlichen Verfassungsbruch anzustreben, indem sie die Religions- und insbesondere die Kultusfreiheit religiöser Minderheiten zu ritzen beabsichtigen würden. ${ }^{17}$ Die Strategie, mit welcher die Verbotsbefürworter diese Vorwürfe zu entkräften versuchten, war beide Male dieselbe: Dem Schächten respektive dem Minarett wurde abgesprochen, religiösen respektive kultischen Charakter zu besitzen. Sie führten an, dass beide nicht auf die heiligen Schriften der jeweiligen Religion zurückzuführen seien. ${ }^{18}$ Der Koran respektive der Tanach und der Talmud wurden von den Verbotsbefürwortern zu Belastungszeugen stilisiert. Die selektive oder gar verfälschende Exegese heiliger Schriften stellt dabei eine klassische Rechtfertigungs- und Rationalisierungsstrategie sowohl des Antisemitismus als auch der Islamfeindlichkeit dar. ${ }^{19}$ Ebenso klassisch wurde dabei gerne auf jüdische und

15 Dies betonen: Krauthammer, Das Schächtverbot (wie Anm. 9), 85-89; Külling, Bei uns (wie Anm. 1), 369-381; Beatrix Mesmer, Das Schächtverbot von 1893, in: Aram Mattioli (Hg.), Antisemitismus in der Schweiz 1848-1960, Zürich 1998, 215-239, 233. Krauthammer, Das Schächtverbot (wie Anm. 9), 87-88. Als Gegenbeispiel kann der Kanton Aargau dienen, wo sowohl medial als auch durch den Tierschutzverein massiv agitiert wurde. Mit rund 90 Prozent sagte der Mittellandkanton überdeutlich ja zum Verbot.

17 Metzger, Antisemitismus (wie Anm. 14), 217-219; Krauthammer, Das Schächtverbot (wie Anm. 9), 48-54; 78-81; Külling, Bei uns (wie Anm. 1), 291; 352-357. Die Gegner eines Schächtverbots waren in ihren Argumentationen indes alles andere als frei von antisemitischen Haltungen.

18 Aus der Fülle an Beispielen: Die Landesväter als Schächtherolde, Beilage zum Tierfreund Nr. 1, 1892; Verus [Andreas Keller-Jäggi], Der «Tiefsittliche» Ernst des Talmud und Schulchan-Aruch. Auch ein Beitrag zur Schächtfrage, o.O. 1893; Unsere Freiheiten bewahren Frauen gegen eine Islamisierung der Schweiz, in: (www.minarett-verbot.ch/downloads/ argumentarium_frauenfreiheit.pdf〉, (21. April 2018); EDU-Solothurn, Flyer 〈Es geht auch ohne Minarette), in: (www.edu-solothurn.ch/pdf/EDU_Flyer_Gegen-Bau-von-Minarette.pdf), (21. April 2018); Interview mit Ulrich Schlüer auf swissinfo.ch, in: «www.swissinfo.ch/ eng/multimedia/anti-minaret-campaigner-puts-case_rightwing-politician-claims-minarets-area-political-symbol/1012760,, 21. April 2018. Im Widerspruch mit dieser Argumentationsstrategie stand der ebenfalls populäre Rache-Diskurs. Das Minarettverbot sei eine Revanche für die Diskriminierung von Christen in islamisch geprägten Ländern. Siehe z.B.: Egerkinger Komitee, Islam-Argumentarium. Minarett, 3, in: (www.minarett-verbot.ch/ downloads/03-minarett.pdf), (21. April 2018).

Siehe: Wetzel, Parallelen (wie Anm. 6), 99-102. 
muslimische «Belastungszeugen〉 zurückgegriffen. ${ }^{20}$ Auf Seiten der Tierschutzvereine wurde beispielsweise bevorzugt auf die Aussagen des einstigen liberaljüdischen Rabbiners Leopold Stein, der sich später zum Atheismus bekannte, verwiesen. ${ }^{21}$

Von Befürwortern des Minarettverbots wurde das Minarett letztlich von einem religiösen zu einem machtpolitischen Symbol umgedeutet und letztlich zum Signum einer angeblich voranschreitenden 〈Islamisierung〉 der Schweiz stilisiert. ${ }^{22}$ Ein Flugblatt der Minarettgegner bringt dies deutlich zum Ausdruck: «In Wahrheit ist ein Verdrängungskampf in Gang. Es geht um Macht. Das Minarett ist Ausdruck eines religiös-politischen Machtanspruchs. Mit Glauben hat es nichts zu tun.» ${ }^{23}$ Christian Waber von der Rechtsaussenpartei EDU und Mitglied des Initiativkomitees, des sogenannten Egerkinger Komitees, ging sogar so weit $\mathrm{zu}$ behaupten, der Islam an sich sei gar keine Religion, sondern eine Kriegserklärung an die christliche und andersgläubige Welt. ${ }^{24}$ Auch den Gegnern des Schächtens war das Konstrukt einer sich hinter dem Deckmantel des Religiösen verbergenden Intention und somit verschwörungstheoretisches Denken eigen. Sie stellten das Schächten als Mittel einer jüdischen «Segregation〉 von der christlichen Gesellschaft dar. ${ }^{25}$ Der Central-Vorstand der deutsch-

20 Siehe etwa: EDU-Solothurn, Flyer (wie Anm. 18); Andreas Keller, Das Schächten der Israeliten, Referat gehalten an einer Versammlung von Thierschutzfreunden am 2. April 1890, Aarau 1890. So auch noch in einer Broschüre gegen das Schächten von 1971: Das sogenannte Schächtverbot. Stellungnahme des Schweizerischen Tierschutzverbandes zum Begehren um Aufhebung von BV Art. 25 ${ }^{\text {bis }}$, Basel 1971, 7.

21 Siehe etwa: Aargauischer Thierschutzverein, Die Schächtfrage in der Schweiz. Ein Wort zur Aufklärung unter spezieller Berücksichtigung der Frage im Aargau, Aarau 1888, 9-11; Keller, Das Schächten (wie Anm. 20), 16-19; Zu Stern siehe: Külling, Bei uns (wie Anm. 1), 291. Die Schächtgegner griffen auch auf verfälschte oder gar gefälschte Gutachten gegen das Schächten zurück: Külling, Bei uns (wie Anm. 1), 296-303.

22 Siehe beispielsweise: Oskar Freysinger, Integration statt Minarett. Ja zum Minarettverbot, in: Schweizerzeit, 9. Oktober 2009; Andreas A. Glarner, Ja zum Minarettverbot!, in: Die Lupe. Informationsbulletin der Aargauischen Vaterländischen Vereinigung, 28,3 (2009), 1-2, 1; Argumentarium «Ja zur Minarettverbots-Initiative», passim, in: (www.minarettverbot.ch/downloads/argumentarium_minarettverbot.pdf), (21. April 2018).

23 Initiativkomitee gegen den Bau von Minaretten, Flyer (Das Minarett und seine Bedeutung), in: (www.minarett-verbot.ch/downloads/flyer-d.pdf), 21. April 2018. Dieselbe Argumentation auch bei einem Plakat des Ostschweizer Komitees, welches ein Minarett zeigt, das die beiden Türme der St. Galler Kathedrale überragt: Keine islamischen Machtsymbole in der Schweiz!, in: (www.tagblatt.ch/ostschweiz/stgallen/kanton/Religionbraucht-keine-Tuerme;art140,1398733), (29. April 2018).

24 Christian Waber, Religiöse [sic!] Frieden der Schweiz gefährdet?, einsehbar unter: «www.minarett-verbot.ch/referate/archiv-2007/religioese-frieden-der-schweiz-gefaehrdet.html), (4. Oktober 2016).

25 Zentral in der Argumentation war die in antisemitischen Kreisen - basierend auf tendenziösen Übersetzungen - populäre Zuschreibung an den Schulchan Aruch, einer frühneuzeitlichen Sammlung jüdischer religiöser Vorschriften, antichristlichen Regelungen zu beinhalten. Die schächtgegnerischen Publikationen der Tierschutzvereine griffen mehrfach darauf zurück. So etwa: Central-Vorstand der deutsch-schweizerischen Tierschutzvereine, Zur Schächtfrage, o.O. 1891; Verus [Andreas Keller-Jäggi], Der «Tiefsittliche» Ernst (wie Anm. 18). 
schweizerischen Tierschutzvereine sprach in diesem Sinne sogar davon, dass nicht die Juden mit Intoleranz konfrontiert seien, sondern vielmehr der Schächtakt einen Ausdruck «krassester Intoleranz gegenüber den Christen» darstelle. ${ }^{26}$

Dass die Genese kultischer Handlungen und Traditionen der Religionen - man denke nur an die Kirchtürme oder die Liturgien der christlichen Kirchen - einen evolutionären Prozess darstellen, wurde von Seiten der Initianten ebenso negiert wie deren zentrale Bedeutung für das religiöse Leben. Die Beschneidung der Kultusfreiheit, die gerade diese Aspekte des religiösen Lebens schützen soll, wurde somit bewusst in Kauf genommen, und die «Mehrheitsgesellschaft» strebte eine asymmetrische Rechtsgrundlage zugunsten der christlichen Konfessionen und zuungunsten der 〈religiös Anderen〉 an. Solche Grundhaltungen sind mit Blick auf die gesellschaftliche Situation der Muslime auch für Deutschland nachweisbar. Eine von der Friedrich-Ebert-Stiftung beauftragte Studie stellte 2010 fest, dass 58,4 Prozent der Befragten die erhebliche Einschränkung der Religionsausübung für Muslime befürworteten. ${ }^{27}$

Die Geringschätzung verfassungsmässig garantierter Grundrechte wurde nicht selten auch durch den populistischen Verweis darauf gerechtfertigt, dass 〈das Volk〉 ein feineres 〈moralisches〉 und 〈sittliches〉 Empfinden besitzen würde als die abstrakt denkende 〈politische Elite〉. Dem 〈Volksgefühl〉 müsse deshalb Genüge getan werden. ${ }^{28}$ Das für ein Schächtverbot agitierende Satiremagazin Der Nebelspalter brachte diesen antielitären Diskurs am Vorabend der Abstimmung sinnbildlich zum Ausdruck. Der Karikaturist verunglimpfte die durch Initiative tangierten Verfassungsrechte als hässliche «Kulturgrazien» und setzte diesen einen kräftig, gesund und aufrecht stehenden gezeichneten Bauern als Personifikation des 〈Volkes〉 entgegen, welcher den Schlachtstier, der zwei schächtende Juden attackiert, von der Leine gelassen hat. ${ }^{29}$

Central-Vorstand der deutsch-schweizerischen Tierschutzvereine, Zur Schächtfrage (wie Anm. 25), 5. Der damalige SVP-Nationalrat Ulrich Schlüer stellte Christentum und Islam einander dichotom gegenüber, indem er ersteres mit Toleranz gleichsetzte und letzterem diese komplett absprach: Interview mit Ulrich Schlüer (wie Anm. 18). Die EDU-Solothurn wiederum betonte, dass durch das Minarettverbot die Glaubens- und Gewissensfreiheit nicht gefährdet, sondern geschützt werde: EDU-Solothurn, Flyer (wie Anm. 18). Oliver Decker/Marliese Weißmann/Johannes Kiess/Elmar Brähler, Die Mitte in der Krise. Rechtsextreme Einstellungen in Deutschland 2010, Bonn 2010, 134, in: 〈library.fes.de/pdffiles/do/07504-20120321.pdf), (21. April 2018).

28 Siehe etwa: Rekursbegründung der Regierungen der Kantone Bern und Aargau gegen den Entscheid des Bundesrathes vom 17. März 1890 betreffend das Schächten der Schlachtthiere an die Bundesversammlung der schweizerischen Eidgenossenschaft, Aarau 1890, 12; Central-Vorstand der deutsch-schweizerischen Tierschutzvereine, Zur Schächtfrage (wie Anm. 25), 6; Interview mit Ulrich Schlüer (wie Anm. 18); Siehe zudem: Külling, Bei uns (wie Anm. 1), 312-314. Bezüglich der Minarettinitiative: Anna Christmann, Die Rolle direktdemokratischer Instrumente in Moschee- und Minarettkonflikten in der Schweiz und in Deutschland, in: Vatter (Hg.), Vom Schächt- zum Minarettverbot (wie Anm. 7), 191-124. Die bedrängten Kulturgrazien, in: Nebelspalter, 19. August 1893. 


\section{Der Islam und das Judentum als 〈rückständige〉 und 〈gewaltbereite〉 Religionen}

Differenzkonstruktion stellte ein zentrales Muster in der Argumentation der Akteure dar, die ein Verbot des Schächtens sowie des Baus von Minaretten propagierten. Während dies bei der Negierung der religiösen Relevanz des Schächtens und des Minaretts noch wenig explizit der Fall war, so war dies bei einem zweiten Diskursbündel umso evidenter. Sowohl in den Abstimmungsdebatten von 1893 als auch jenen von 2009 stellte die Konstruktion der Dichotomie 〈rückständige〉〈fremde〉 vs. 〈modern-aufgeklärte〉 eigene〉 Kultur ein wiederkehrendes Diskursmuster dar. ${ }^{30}$ Mit Blick auf die islamfeindliche Argumentation zeigt sich nicht zuletzt, wie stark Religion von den Minarettgegnern kulturalistisch gedacht und als Mittel zur Hierarchisierung eingesetzt wurde. ${ }^{31}$ So sprach etwa das Egerkinger Komitee in seinem Argumentarium zur Minarettverbotsinitiative davon, dass es im Falle des Islam weniger um Religion denn um Zivilisation gehe. ${ }^{32}$ In der kulturellen Herabminderung des von ihnen monolithisch verstandenen Islam und mit ihm grosser Regionen des Mittleren Ostens und Nordafrikas werden dabei festetablierte Topoi der Islamfeindlichkeit sichtbar, die in kolonialen Sichtweisen vergangener Jahrhunderte wurzeln. ${ }^{33}$ Gerade auch der ausgerechnet von den wertkonservativen Initianten vorgebrachte Vorwurf des angeblich rückständigen Frauenbildes des Islam weist eine lange Tradition auf. ${ }^{34}$ In der Abstimmungskampagne von 2009 gehörten Assoziierungen 〈der Muslime〉 und gerade auch der muslimischen Immigranten mit Gewaltbereitschaft, Terrorismus, Radikalismus und Fundamentalismus zum Grundstock

Besonders stark in: Argumentarium «Ja zur Minarettverbots-Initiative» (wie Anm. 22), passim. Zudem: Keller, Das Schächten (wie Anm. 20), 22; Central-Vorstand der deutschschweizerischen Tierschutzvereine, Zur Schächtfrage (wie Anm. 25), 3; 5; Lukas Reimann, Kaida und Co. sind längst in der Schweiz angekommen, in: Neue Zürcher Zeitung, 6. November 2009, in: 〈www.nzz.ch/kaida_und_co_sind_laengst_in_der_schweiz_angekommen-1.3972013), (22. April 2018). Die Diskursmuster greifen dabei auf altetablierte antisemitische respektive islamophobe Stereotypisierungen zurück. $\mathrm{Zu}$ den Mechanismen von Stereotypen - gerade auch mit Blick auf die bei Heterostereotypen unweigerlich implizit mitzudenkenden Autostereotypen - siehe etwa: Hans Henning Hahn, 12 Thesen zur Stereotypenforschung, in: ders./Elena Mannová (Hg.), Nationale Wahrnehmungen und ihre Stereotypisierung. Beiträge zur Historischen Stereotypenforschung, Frankfurt a.M. 2007, $15-24$.

31 In der Forschung zur Islamfeindlichkeit wird zurecht die diskursive Integration neorassistischer Denkmuster betont. Siehe besonders: Naime Cakir, Islamfeindlichkeit. Anatomie eines Feindbildes in Deutschland, Bielefeld 2014; Yasemin Shooman, "....weil ihre Kultur so ist». Narrative des antimuslimischen Rassismus, Bielefeld 2014.

32 Argumentarium «Ja zur Minarettverbots-Initiative» (wie Anm. 22), 2. Siehe zudem auch: Egerkinger Komitee, Islam-Argumentarium - Grundbegriffe, in: (www.minarett-verbot.ch/downloads/argumentarium-komplett-d.pdf), (22. April 2018).

33 Siehe etwa: Chris Allen, Islamophobia, Farnham/Burlington 2010, 25-35; Wolfgang Benz, Die Feinde aus dem Morgenland. Wie die Angst vor den Muslimen unsere Demokratie gefährdet, München 2012, 49-60; Shooman, «...weil ihre Kultur so ist» (wie Anm. 31), 35-53.

Shooman, «...weil ihre Kultur so ist» (wie Anm. 31), 76-79. Quellenbeispiele: Glarner, Ja zum Minarettverbot! (wie Anm. 22); Unsere Freiheiten (wie Anm. 18). 
des Argumentariums. ${ }^{35}$ Der Aargauer Grossrat Andreas Glarner, am rechten Rand der SVP politisierend, bemerkte suggestiv: «Natürlich ist nicht jeder Muslime ein Terrorist - aber wenn man sich die letzten grossen Anschläge so überlegt, kommt man zum Schluss, dass fast jeder Terrorist ein Muslime war.» ${ }^{36}$ Das wiederholt an die Decke gemalte Szenario der Gefahr einer Islamisierung Europas und potenziell auch der Schweiz kann ebenfalls diesem diskursiven Feld zugeordnet werden, wird doch einer drohenden Invasion das Wort geredet. ${ }^{37}$

Die in der Anti-Schächt-Kampagne zum argumentativen Grundstock gehörenden Rückständigkeits-Diskurse zeigten sich anschlussfähig für verschiedene antisemitische Topoi. So wurde〈den Juden〉 eine grundsätzliche Grausamkeit vorgeworfen, die sich letztlich nicht nur gegenüber dem Tier - in Form von Tierquälerei $^{38}$-, sondern auch im Umgang mit nichtjüdischen Menschen zeigen würde. ${ }^{39}$ Besonders frappant unterstellte Samuel Kurth ${ }^{40}$ in der sich stark für das

Zur Assoziation mit Gewaltbereitschaft und Terrorismus besonders: Egerkinger Komitee, Islam-Argumentarium - Grundbegriffe (wie Anm. 32), passim. Zum Fundamentalismusvorwurf u.a.: Argumentarium «Ja zur Minarettverbots-Initiative» (wie Anm. 22), passim; Reimann, Kaida (wie Anm. 30). Gerade auch das umstrittene Anti-Minarett-Plakat förderte Assoziationen mit Terror, da die Minarette, welche auf der Schweizer Fahne stehen, Ähnlichkeiten mit Raketen aufweisen: Plakat 〈Ja zum Minarettverbot), in: (www.minarettverbot.ch/grafiken/plakat-d.pdf), (25. April 2018). Eine Medienanalyse bestätigt eine diesbezügliche Akzentuierung vorab in den Deutschschweizer Medien: Patrick Ettinger, Muslime in den Medien zunehmend problematisiert. Studie zur Qualität der Berichterstattung über Muslime in der Schweiz, in: Muslimfeindlichkeit: Gesellschaft, Medien und Politik. Tagungsakten der EKR - 11.09.2017, 69-75.

36 Das Zitat entstammt einer Rede Glarners am kantonalen Parteitag und folgte als Zitat seinem Artikel «Ja zum Minarettverbot» im Informationsbulletin der Aargauischen Vaterländischen Vereinigung: Es sagte Andreas Glarner, in: Die Lupe. Informationsbulletin der Aargauischen Vaterländischen Vereinigung, 28,3 (2009), 3.

37 Den Islamisierungs-Diskurs bedienen etwa: Walter Wobmann, Entwicklung der Parallelgesellschaften unsere Regeln gelten für alle [sic!], in: 〈www.walter-wobmann.ch/aktuell/svpartikel/entwicklung-der-parallelgesellschaften/s, (29. April 2018); Ulrich Schlüer, Ja zur Minarettverbots-Initiative. Ein Abstimmungskampf mit beunruhigenden Merkmalen, in: «schluer.ch/aktuell/presseartikel/archiv-2009/ja-zur-minarettverbots-initiative.html), (29. April 2018); Reimann, Kaida (wie Anm. 30). Dieser Aspekt lässt sich in alte Tradititonslinien der Islamfeindlichkeit einordnen, die die Beziehung zwischen dem «christlichen Okzident) und dem (islamischen Orient) als Konfliktgeschichte beschreiben. Siehe hierzu etwa: Benz, Die Feinde (wie Anm. 33), 49-60; Gerdien Jonker, Europäische Erzählmuster über den Islam. Wie alte Feindbilder in Geschichtsschulbüchern die Generationen überdauern, in: Thorsten Gerald Schneiders (Hg.), Islamfeindlichkeit. Wenn die Grenzen der Kritik verschwimmen, Wiesbaden 2009, 71-83.

Insbesondere gehörten dramatisierende und brutalisierende Schilderungen des Schächtvorgangs zum Standardrepertoire: Keller, Das Schächten (wie Anm. 20); Die Landesväter (wie Anm. 18), 7-8; Wie es beim Schächten zu- und hergeht?, in: Berner Volkszeitung, 12. August 1893; Ein Gespräch über das eidgenössische Schächtverbot, in: Christlicher Volksbote, 16. August 1893, 259-261, 260.

39 Siehe z.B.: Central-Vorstand der deutsch-schweizerischen Tierschutzvereine, Zur Schächtfrage (wie Anm. 25), 3-4; Otto Zellweger, Nachrichten, in: Appenzeller Sonntagsblatt, 19. Dezember 1891, 407-408, 407; Stanislaus an Ladislaus, in: Nebelspalter, 12. August 1893; Ulrich Dürrenmatt, Juden haben kein Erbarmen, in: Berner Volkszeitung, 19. August 1893. 
Schächtverbot einsetzenden konservativen Berner Volkszeitung Inhumanität, indem er bilanziert, dass das Schächten das Abbild des jüdischen Treibens unter den anderen Völkern darstelle, und in einer Beilage zum Thierfreund wurde 1892 an die Bauern appelliert, ihr Schlachtvieh nur den Händen eines «menschlichen Schlächters» zu übergeben. Auch der Karikaturist und Künstler Fritz Boscovits zeichnete «den schächtenden Juden〉 als Menschenfeind. ${ }^{41}$ Selbst Autoren, die sich im Sinne der Religions- und Kultusfreiheit gegen die Schächtverbotsiniatiative stellten, griffen auf diese antisemitischen Feindbilder zurück, so etwa Pfarrer Robert Ischer in den liberalprotestantischen Schweizerischen Reformblättern:

«Wäre das Schächten nicht zufällig eine Sitte der Juden, so wäre gegen diese Schlachtart sicherlich nicht so bald Klage geführt worden. Nicht ihre Tier-, sondern ihre Menschenquälerei ist es, welche am meisten ärgert, ihr herzloser Wucher, ihre Aufdringlichkeit und systematische Ausbeutung der Mitbürger im Handelsverkehr.» ${ }^{42}$

\section{Beharrung auf Statusdifferenz}

Auch das dritte Diskursbündel konstruiert Differenz. Es stilisiert 〈die Juden〉 und «die Muslime〉 zu Fremdgruppen und beinhaltet Topoi, die eine rechtliche und gesellschaftliche Statusdifferenz zwischen Mehrheit und Minderheit fordern. So wurden das Schächten und das Minarett zu Handlungen respektive Symbolen erklärt, die angeblich 〈Sonderrechte〉 darstellen und Juden und Muslime gegenüber der imaginierten 〈Mehrheitsgesellschaft〉 Vorteile verschaffen würden. ${ }^{43}$ «Gleiches Recht für alle Bürger unseres Landes!», so postulierte dies Andreas Keller-Jäggi, Präsident des Aargauischen Tierschutzvereins und einer der zen-

Siehe auch die Beispiele - teilweise mit Ritualmordvorwürfen kombiniert - bei: Krauthammer, Das Schächtverbot (wie Anm. 9), 63-73.

Samuel Kurth, Israel kennt kein Erbarmen, in: Berner Volkszeitung, 19. August 1893.

Fritz Boscovits, Der Hofmetzger an der Arbeit, in: Nebelspalter, 10. Juni 1893.

42 Robert Ischer,

43 Siehe beispie Zur Schächtfrage (wie Anm. 25), 7; Keller, Das Schächten (wie Anm. 20), 25. Siehe auch die Beispiele in: Krauthammer, Das Schächtverbot (wie Anm. 9), 90; Külling, Bei uns (wie Anm. 1), 304; Walter Wobmann, Wie kam es zur Minarett-Verbots-Initiative, in: (www.walter-wobmann.ch/aktuell/svp-artikel/wie-kam-es-zur-minarett-verbots-initiative), (29. April 2018); Patrick Freudiger, Kurz-Argumentarium zur Minarettverbots-Initiative, 3-5, in: 〈www.minarett-verbot.ch/mobile/smartphone/downloads/kurz-argumentarium_minarettverbot.pdf), (29. April 2018). Der Sonderrechts-Diskurs trat in der Anti-MinarettKampagne häufig in Kombination mit weiteren rechtlichen Fragen des Zusammenlebens auf wie etwa dem Schulschwimmen oder muslimischen Grabfeldern. Siehe etwa: Aline Indergard, Leserbrief 〈Toleranzgrenze〉, in: St. Galler Tagblatt, 11. September 2009; Freysinger, Integration (wie Anm. 22); Reimann, Kaida (wie Anm. 30). 
tralen Akteure in der Anti-Schächt-Kampagne. ${ }^{44}$ Diese diskriminierende Optik negiert die Tatsache, dass sich ganz im Gegenteil die Mehrheit gegenüber Minderheiten Vorrechte ausbedingt, indem nur ihre kultischen Handlungen und Symbole uneingeschränkt ausgeübt werden respektive präsent sein dürfen. Besonders evident wurde dies beim Kampf gegen den Bau weiterer Minarette - seit den 1960er Jahren waren deren vier in der Schweiz errichtet worden ${ }^{45}$-, denn bei diesen ging es um die Frage der Sichtbarkeit einer nichtchristlichen Religion im öffentlichen Raum. Während 〈Hinterhofmoscheen〉 toleriert wurden, sollten mit dem Minarettverbot nicht zuletzt repräsentative Bauten verhindert werden, da diese den Eindruck vermitteln würden, dass der Islam in der Schweiz ‘angekommen> sei und die Muslime auch in der Schweiz bleiben möchten. ${ }^{46}$ Zugleich wurden, wie bereits ausgeführt, der religiöse Charakter von Minaretten negiert und diese stattdessen zu Machtsymbolen und somit zu Instrumenten einer «Islamisierung> erklärt. ${ }^{47}$ Ikonographisch markant brachte dies das über die schweizerischen Landesgrenzen hinaus bekannt gewordene Kampagnen-Plakat aus dem Umfeld der SVP zum Ausdruck. Neben den auf der Schweizer Flagge stehenden Minaretten war es insbesondere die vollverschleierte Muslima, die dieses Feindbild bediente, stand sie doch in keinem direkten Zusammenhang mit der Sachfrage des Baus von Minaretten. ${ }^{48}$ Einen noch expliziteren Ansatz wählte das Ja-Komitee des Kantons Luzern. Auf deren Plakat prangte «Islamisierung stoppen! Minarettverbot Ja». ${ }^{49}$ Ähnliche Kritik löste im letzten Drittel des 19. Jahrhunderts der Bau von Synagogen in Schweizer Ortschaften aus. ${ }^{50}$ Öffentliche Sichtbarkeit nichtchristlicher Religionen hatte es in der Schweiz seit 1848 generell schwer, ${ }^{51}$ doch der Blick auf Langenthal im Kanton Bern bezeugt - klare islamfeindliche Vorurteilsstrukturen offenlegend -, dass heute primär die muslimischen Gemeinden auf Widerstand stossen. Es war mit das Gesuch

44

Siehe: Felix Müller/Mathias Tanner, Muslime, Minarette und die Minarett-Initiative in der Schweiz, in: dies./Frank Mathwig/Wolfgang Lienemann (Hg.), Streit um das Minarett. Zusammenleben in der religiös pluralistischen Gesellschaft, Zürich 2009, 21-43, 32-39. Diese wurden errichtet in: Zürich (1963), Genf (1978), Winterthur (2005) und Wangen bei Olten (2009).

46 Siehe etwa: Egerkinger Komitee, Islam-Argumentarium - Grundbegriffe (wie Anm. 32), 11; Argumentarium «Ja zur Minarettverbots-Initiative» (wie Anm. 22), 2; Wobmann, Entwicklung (wie Anm. 37). Zur Präsenz von Religion im öffentlichen Raum und deren Symbolhaftigkeit: Martin Baumann/Andreas Tunger-Zanetti, Streit um Symbole im öffentlichen Raum. Bauten «fremder» Religionsgemeinschaften in der Schweiz, in: Neue Zürcher Zeitung, 1. Oktober 2009.

47 Siehe die Nachweise in Anm. 22.

48 Plakat 〈Ja Zum Minarettverbot〉 (wie Anm. 35).

49 Plakat 〈Islamisierung Stoppen!〉, in:〈www.swissinfo.ch/ger/minarettverbot/590418〉, (29. April 2018).

50 Metzger, Antisemitismus (wie Anm. 14), 193-194; Ron Epstein-Mil, Die Synagogen der Schweiz. Bauten zwischen Emanzipation und Akkulturation, Zürich 2008, 56-58.

51 Siehe hierzu: Baumann/Tunger-Zanetti, Streit (wie Anm. 46). 
um die Errichtung eines Minarettes in dieser Landstadt, das die Initiative losgetreten hatte. Die gleichzeitige Errichtung eines Sikh-Tempels 2008 hingegen war auf keinen Widerstand gestossen. ${ }^{52}$ In der Ablehnung muslimischer Symbole in der Öffentlichkeit wird ein christlicher Superioritätsanspruch der Initiativbefürworter gegenüber dem Islam ersichtlich. Sowohl der jüdischen als auch der muslimischen Minderheit wurde beziehungsweise wird somit letztlich ein Leben am Rande der Gesellschaft zugedacht und eine Unterordnung unter die «Mehrheitsgesellschaft〉 ohne Einforderung gleicher Rechte im Bereich des Religiösen und Kultischen verlangt.

Die Forderung nach rechtlicher Statusdifferenz und letztlich nach der $\mathrm{Zu}$ rückstufung von Juden und Muslimen auf (Fremdgruppen〉 minderen Rechtes mündete sowohl in der Abstimmung von 1893 als auch jener von 2009 in Aussagen, die Juden und Muslime lediglich einen Gast-Status in der Schweiz zuwiesen. ${ }^{53}$ Walter Wobmann, Solothurner Nationalrat der $S V P$ und einer der zentralen Akteure im Initiativkomitee, brachte dies mit Blick auf den Abstimmungsausgang deutlich zum Ausdruck und stellte zugleich den Vorwurf einer angeblich ungenügenden Integration in den Raum:

«Fakt ist [...], dass der Islam hier nur zu Gast ist. Zu Gast in einem christlichen und zutiefst demokratischen Land. Wie von allen ausländischen Gästen, erwarten wir auch von Muslimen eine rasche Integration, also die Anerkennung sowohl unserer Gesetze als auch unserer ungeschriebenen Regeln [...].. ${ }^{54}$

Die Schweizer sollten - im Sinne solcher Stimmen - Herren im eigenen Haus bleiben. Eine Formulierung, die gerade in der Schächtdebatte von 1893 wiederholt fiel. ${ }^{55}$

\section{Stigmatisierung als 〈Fremde〉}

Explizite Fremdheitszuschreibungen stellen ein letztes gemeinsames Diskursbündel dar, welches die Analyse der Argumentationsstrukturen der Befürworter des Schächt- und Minarettverbots aufzeigt. Sowohl Juden als auch Muslime wurden letztlich mit Blick auf ihre Religion als auch auf ihre Kultur und Herkunft oft als doppelt 〈fremd〉 charakterisiert, wobei in der Differenzkonstruktion

52 Humanyun Ansari/Farid Hafez, Islamophobia: An Introduction, in: dies. (Hg.), From the Far Right to the Mainstream. Islamophobia in Party Politics and the Media, Frankfurt/New York 2012, 7-28, 7.

Siehe z.B.: Die Landesväter (wie Anm. 18); Ein Gespräch (wie Anm. 38), 260; Argumentarium «Ja zur Minarettverbots-Initiative» (wie Anm. 22), 3. Mit einem allgemeinen Blick auf Minarett-Debatten: Wolfang Lienemann, Argumente für ein Minarett-Verbot? Eine kritische Analyse, in: Tanner/Müller/Mathwig/Lienemann (Hg.), Streit um das Minarett (wie Anm. 45), 123-139, 133.

54 Wobmann, Wie kam es (wie Anm. 43).

55 Siehe etwa: Die Landesväter (wie Anm. 18); Ein Gespräch (wie Anm. 38), 260. 
Religion und Kultur nicht selten in essentialisierender Weise verschmolzen. ${ }^{56}$ Wiederholt schlägt dabei durch, dass sowohl Juden wie Muslime als <aussereuropäische Fremde> konstruiert werden ${ }^{57}$ So schreibt der populistisch agierende konservativ-protestantische Redaktor und Politiker Ulrich Dürrenmatt am 19. August 1893 - unmittelbar vor der Abstimmung über das Schächtverbot - in seinem Gedicht «Juden haben kein Erbarmen» vom 〈Juden〉 als dem «Fremdling aus dem Osten». ${ }^{58}$ Gerade mit Blick auf den Islam stellt die dichotome Identitätskonstruktion 〈Abendland-Morgenland〉 historisch gesehen einen sehr bedeutenden islamfeindlichen Diskurs dar. ${ }^{59}$ So wurde und wird auf der Website des Initiativkomitees von den Muslimen als religiöser Minderheit gefordert, sich an die «christlich-abendländisch geprägte Bevölkerung und Kultur» anzupassen und erst recht deshalb auf den Bau von Minaretten zu verzichten. ${ }^{60}$

Beide Abstimmungskampagnen sind letztlich gerade auch migrationspolitisch zu verstehen. Durch eine rechtliche und gesellschaftliche Zurücksetzung sollte die Schweiz unattraktiv für eine fortdauernde jüdische und islamische Einwanderung gemacht werden. ${ }^{61}$ Besonders im Falle der Antiminarettinitiative stellte Migration denn auch ein wiederkehrendes Stichwort dar. So wurde etwa auf den seit den 1980er Jahren stark angestiegenen muslimischen Bevölkerungs-

Siehe z.B.: Dürrenmatt, Juden haben kein Erbarmen (wie Anm. 39); EDU-Solothurn, Flyer (wie Anm. 18); Egerkinger Komitee, Islam-Argumentarium - Grundbegriffe (wie Anm. 32), 7-9. In essentialisierender Weise findet sich diese Argumentation vorab bei den Minarett-Gegnern. Siehe zur Fremdheitskonstruktion in der Islamfeindlichkeit: Marco Guini/ Miruna Morariu, Intolerance Begets Intolerance: Explaining Negative Attitudes Towards Foreigners and Muslims In Switzerland, 1996-2007, in: Simon Hug/Hanspeter Kriesi (Hg.). Value Change In Switzerland, Lanham 2010, 91-97, 95; Shooman, «...weil ihre Kultur so ist» (wie Anm. 31), 45-51.

57 Siehe etwa: Central-Vorstand der deutsch-schweizerischen Tierschutzvereine, Zur Schächtfrage (wie Anm. 25), 3; Samuel Kurth, Der Aufruf des sogenannten Schächtkomitees, in: Berner Volkszeitung, 12. August 1893. Siehe auch: Külling, Bei uns (wie Anm. 1), 73-76. Bezüglich der Wahrnehmung des Islam in der Schweiz: Samuel M. Behloul, Religion und Religionszugehörigkeit im Spannungsfeld von normativer Exklusion und zivilgesellschaftlichem Bekenntnis. Islam und Muslime als öffentliches Thema in der Schweiz, in: Brigit Allenbach/Martin Sökefeld (Hg.), Muslime in der Schweiz, Zürich 2010, 43-65, 47. Dürrenmatt, Juden haben kein Erbarmen (wie Anm. 39).

59 Siehe z.B.: Daniel Bax, Angst ums Abendland. Warum wir uns nicht vor Muslimen, sondern vor den Islamfeinden fürchten sollten, Frankfurt a.M. 2015, 37-46; Wolfgang Benz, Ansturm auf das Abendland? Zur Wahrnehmung des Islam in der westlichen Gesellschaft, Wien 2013, 23-30; Jonker, Europäische Erzählmuster (wie Anm. 37).

61 Bezüglich der Schächtverbotsinitiative betont dies v.a.: Mesmer, Das Schächtverbot (wie Anm. 15), 234. Zur Vermischung mit der Einwanderungs-Thematik im Falle der AntiMinarett-Initiative: Samuel M. Behloul, Minarett-Initiative. Im Spannungsfeld zwischen Abwehr-Reflex und impliziter Anerkennung neuer gesellschaftlicher Fakten, in: Tanner/ Müller/Mathwig/Lienemann (Hg.), Streit um das Minarett (wie Anm. 45), 103-122, 119. 
anteil verwiesen. ${ }^{62}$ Damit wurde ein Thema aufgegriffen, das die SVP schon länger 〈bewirtschaftete〉. Einwanderung - so extrapolierten Abstimmungskomitees aus dem Umfeld der Partei in Inseraten anlässlich mehrerer Abstimmungsvorlagen zu Migrationsthemen - würde zu einem Einflussgewinn, ja zu einer Dominanz 〈der Muslime〉 in der Schweiz führen. ${ }^{63}$ Eines der Inserate sprach sich implizit gegen die Einbürgerung von Muslimen aus. ${ }^{64}$ Eine kritische Haltung gegen deren Einbürgerung schien auch im Argumentarium des Initiativkomitees für das Minarettverbot auf. ${ }^{65}$ Der in der Schweiz seit über hundert Jahren in der Politik präsente fremdenfeindliche Überfremdungsdiskurs wurde in der Antiminarettabstimmung in einen Islamisierungsdiskurs umgegossen. Fast schon ins Apokalyptische steigern sich dabei die Ausführungen von Lukas Reimann, St. Galler Nationalrat der SVP, der die Antiminarettinitiative zur schweizerischen und europäischen Nagelprobe erklärt:

«Wenn wir die menschenrechtswidrigen, antidemokratischen und reaktionärpatriarchalischen Teile des Islam nicht endlich laut und deutlich anprangern, wenn wir für islamische Einwanderer nicht ganz klare Regeln schaffen und von ihnen Integration sowie Akzeptanz unserer Werte gesetzlich einfordern, wenn wir nicht laut und entschlossen Ideen wie jene von Minarett-Bauten im Keime ersticken, werden wir die Errungenschaften von zig Generationen zerstören, Europa um Jahrhunderte zurückwerfen und unsere Freiheit verlieren.. ${ }^{66}$

Eine absolute Integrations- respektive Anpassungsforderung entsprach einem populären Argumentationsmuster im Abstimmungskampf. ${ }^{67}$ Darin konnten mehr oder minder explizite Aufforderungen an Muslime eingearbeitet sein, bei dieser Forderung zuwiderlaufenden Interessen die Schweiz wieder zu verlassen. ${ }^{68}$

Siehe: Reimann, Kaida (wie Anm. 30); Unsere Freiheiten (wie Anm. 18), 1; Wobmann, Entwicklung (wie Anm. 37).

63 Die entsprechenden Inserate wurden von Akteuren geschaltet, die in zentraler Weise auch in die Lancierung der Minarettverbotsinitiative involviert waren. Sie wurden anlässlich der Kampagne gegen die erleichterte Einbürgerung 2004 sowie der Abstimmung über die Masseneinwanderungsinitiative 2014 gedruckt: Überparteiliches Komitee gegen Masseneinbürgerungen, Inserat 〈Muslime bald in der Mehrheit〉, in: 〈www.20min.ch/schweiz/news/story/SVP--16-Millionen-Einwohner-bis-2060-17503744\#showid=89550\&index=1), (3. Mai 2018); Egerkinger-Komitee, Inserat 〈Bald 1 Million Muslime?), in: (www.blick.ch/news/ politik/link-auf-der-homepage-steckt-doch-die-svp-hinter-dem-burka-inserat-id2659629.html, (3. Mai 2018). Siehe diesbezüglich auch: Georg Kreis, Zur Islamophobie in der schweizerischen Variante, in: ders., Vorgeschichten zur Gegenwart. Ausgewählte Aufsätze, Bd. 5, Basel 2011, 477-495.

Überparteiliches Komitee gegen Masseneinbürgerungen, Inserat (wie Anm. 63). Egerkinger Komitee, Islam-Argumentarium - Grundbegriffe (wie Anm. 32), 5-6; 30.

Lukas Reimann, Aufklären statt verschleiern, In Students.ch, in: (www.students.ch/magazin/details/28435/Minarett-Debatte-Aufklaeren-statt-vers), (4. Mai 2018).

67 Siehe z.B.: Wobmann, Wie kam es (wie Anm. 43); Unsere Freiheiten (wie Anm. 18), 3. In der Schächtverbotskampagne enthielt die Hausherr/Wirt-Metapher ebenfalls diese Konnotation. Siehe hierfür weiter oben.

Siehe etwa: Reimann, Kaida (wie Anm. 30); Egerkinger Komitee, Islam-Argumentarium Grundbegriffe (wie Anm. 32), 6. Das Argument tauchte wiederholt auch in Leserbriefen 


\section{Diskriminierung durch direkte Demokratie?}

Dass die gegen religiöse Minderheiten gerichteten Schächt- und Minarettverbotsinitiativen an der Urne auf Zustimmung stiessen, beweist die Wirkmächtigkeit antisemitischer und islamfeindlicher und der mit diesen verknüpften fremdenfeindlichen Diskurse. Die Lancierung und die Annahme der beiden Volksinitiativen zielten auf die Ausgrenzung der Juden und der Muslime als 〈Fremde〉 aus der von den Initianten letztlich (christlich〉 verstandenen schweizerischen Gesellschaft und somit auf die Schaffung von Statusdifferenz ab. Religiöse und kulturelle Topoi verdichteten sich nicht selten in einer essentialisierenden Stossrichtung. Das direktdemokratische Instrument der Volksinitiative zeigt sich in diesen beiden Fällen somit als mehrheitspolitisches Instrument, das zur Diffamierung und Zurücksetzung von Minderheiten beitragen kann. Die Gleichberechtigung kann somit für Minderheiten prekär bleiben. Das Schächten und der Bau von Minaretten wurden, dies zeigen die Aussagen der Verbotsbefürworter deutlich, nicht als isolierte politische Fragen aufgegriffen, sondern die Initiativen richteten sich im Endeffekt pauschal gegen die Juden und Muslime in der Schweiz und potentielle jüdische und muslimische Immigranten. Die Argumente der Initiativgegner, die nicht Teil der vorliegenden Analyse waren, zeigten sich hingegen in ihrer stark verfassungsrechtlichen Ausrichtung gegenüber diesen Pauschalisierungen in beiden Abstimmungen als nicht mehrheitsfähig. ${ }^{69}$

Die Analyse der beiden Volksabstimmungen gewährt auch einen Einblick in das Nationsverständnis der schweizerischen Bevölkerung. Im Gegensatz zum oft tradierten, idealisierten Selbstbild der Schweiz als multikulturelle Willensnation dominierte und dominiert in breiten Bevölkerungskreisen ein kulturelles, ja kulturalistisches Nationsverständnis. Der Multikulturalismus der Schweiz wird exklusiv gedacht und bleibt in dieser Identitätskonstruktion auf die <indigenen> (Sprach-)Kulturen beschränkt. ${ }^{70}$

auf, so etwa: Roswitha Mastel-Göbel, Leserbrief (Land so erhalten, wie es ist), in: St. Galler Tagblatt, 9. Oktober 2009.

69

Für die Argumentationsstrategien der Initiativgegner siehe: Vatter/Milic/Hirter, Stimmverhalten (wie Anm. 11), 160-169; Christmann/Danaci/Krömler, Ein Sonderfall? (wie Anm. 11), 179-185; Külling, Bei uns (wie Anm. 1), 314-316. Im Unterschied zu den Schächtverbotsgegnern, die sehr oft selbst antisemitische Topoi in ihre Argumentation integrierten, scheinen - so eine These, die durch eine ausgedehnte Analyse zu erhärten wäre - in der Abstimmung über das Verbot des Baus von Minaretten islamophobe Muster in den ablehnenden Stellungnahmen kaum präsent gewesen zu sein.

Für die These des exklusiven Multikulturalismus siehe: Damir Skenderovic, Constructing Boundaries in a Multicultural Nation: The Discourse of (Overforeignization) in Switzerland, in: Rainer Ohliger/Karen Schönwälder/Triadafilos Triadafilopoulos (Hg.), European Encounters. Migrants, Migration and European Societies since 1945, Aldershot 2003, 186209, 200-201. 
Konstruktion von Differenz: Ein Vergleich argumentativer Strategien der Befürworter der Schächtverbotsinitiative (1893) und der Antiminarettinitiative (2009)

Durch das direktdemokratische Instrument der Volksinitiative sind 1893 mit dem Schächtverbot und 2009 mit dem Minarettverbot zwei Artikel in die Bundesverfassung aufgenommen worden, welche die Juden und Muslime gegenüber der christlichen Mehrheit in der Schweizer Bevölkerung diskriminieren. Im Zentrum der Untersuchung stehen die Parallelen in den Argumentationsstrategien der Befürworter der beiden Verbotsvorlagen. So wurde auf einer klaren Statusdifferenz zwischen (christlicher Mehrheit) und «nichtchristlicher Minderheit) beharrt sowie dem Minarett als auch dem Schächten die religiöse Relevanz abgesprochen. $\mathrm{Zu}$ den argumentativen Parallelen gehörte zudem die Konstruktion von Differenz, indem der Islam und das Judentum beispielsweise als 〈rückständig〉 und 〈gewaltbereit〉 und letztlich als grundsätzlich 〈fremd〉 diffamiert wurden.

Antisemitismus - Islamfeindlichkeit - Fremdenfeindlichkeit, Differenzkonstruktion konfessionelle Ausnahmeartikel - Schächtverbot - Minarettverbot - direkte Demokratie.

\section{Construction de différences: Comparaison des stratégies argumentatives des partisans de l'initiative contre l'abattage rituel (1893) et de l'initiative anti-minarets (2009)}

En faisant usage de l'initiative populaire, instrument de démocratie directe, deux articles discriminant les juifs et les musulmans par rapport à la majorité chrétienne ont été inscrits à la Constitution fédérale: d'une part, l'interdiction de l'abattage rituel en 1893, d'autre part, l'interdiction de minarets en 2009. Cette analyse porte sur les parallèles entre les stratégies argumentatives des partisans des deux initiatives. On insista sur une distinction de statut claire entre la «majorité chrétienne〉 et la «minorité non-chrétienne». De plus, on contesta la pertinence religieuse des minarets et de l'abattage rituel. La construction de différences fit également partie intégrante des argumentaires, par exemple au vu du fait que l'islam et le judaïsme furent considérés comme 〈attardés〉, 〈enclins à la violence et, d'une manière générale, ‘étrangers'.

Antisémitisme - islamophobie - xénophobie, construction de différences - article d'exception confessionnelle - interdiction de l'abattage rituel - interdiction des minarets démocratie directe.

\section{Costruzione di differenze: un confronto di strategie argomentative dei sostenitori delle iniziative sul divieto della macellazione rituale (1893) e contro l'edificazione di minareti (2009)}

Attraverso lo strumento democratico dell'iniziativa popolare sono stati inseriti nella Costituzione federale nel 1893 il divieto della macellazione rituale e nel 2009 il divieto di edificare dei minareti. Questi due articoli discriminano gli ebrei e i musulmani rispetto alla maggioranza cristiana nella popolazione svizzera. Al centro della ricerca stanno i parallelismi nelle stategie argomentative dei sostenitori dei due divieti. Mentre s'insisteva su una chiara differenza di statuto tra la «maggioranza cristiana» e la "minoranza noncristiana», veniva negata una rilevanza religiosa riguardo ai minareti e alla macellazione. Tra i parallelismi argomentativi c'era anche la costruzione di differenze, in quanto per esempio l'Islam e il Giudaismo erano indicati come "arretrati», "violenti» e infine fondamentalmente «estranei».

Antisemitismo - Inimicizia nei confronti dell'Islam - Inimicizia nei confronti degli stranieri - costruzione di differenze - Articoli confessionali d'eccezione - Divieto della macellazione rituale - Divieto d'edificazione di minareti - Democrazia diretta. 
Construction of difference: A comparison of argumentative strategies between the adherents of the ritual slaughter (1893) and the anti-minaret initiative (2009)

With the ban on ritual slaughter in 1893 and the ban on new minarets in 2009, two popular initiatives were accepted by the Swiss voters resulting in a discrimination of the Jews and Muslims respectively in Switzerland. The article analyzes the parallels existing amongst the argumentation strategies of the supporters of the two popular initiatives. They persisted on a difference in the status between the "Christian majority" and the «non-Christian minorities» while also denying the religious relevance of both ritual slaughter and minarets. Furthermore, constructing differences was a key common to both argumentations. In doing so, Islam and Judaism were, for example, disparaged as «backward» and «violent», and ultimately as fundamentally as «alien».

Anti-Semitism - Islamophobia - xenophobia - construction of difference - articles of exception - ban on ritual slaughter - ban on minarets - direct democracy.

Thomas Metzger, Prof. Dr., Pädagogische Hochschule St. Gallen. 
